\title{
Síndrome de Fournier Secundária a Adenocarcinoma de Próstata Avançado: Relato de Caso
}

\author{
Fournier's Syndrome Secondary to Advanced Prostatic Adenocarcinoma: Case Report
}

\author{
RODRIGO ROCHABATISTA ${ }^{1}$; PAULO ROBERTO RAMACCIOTTIFILHO'; CARLOS ALBERTO \\ TORRES DE CASTRO ${ }^{1}$; MARCUS FÁBIO MAGALHÃES FONSECA²; IDBLANCARVALHO DEALBUQUERQUE ${ }^{2}$; \\ GALDINO JOSÉ SITONIOFORMIGA ${ }^{3}$
}

\begin{abstract}
1. Residente de Coloproctologia do Hospital Heliópolis; 2. Médico Assistente do Serviço de Coloproctologia do Hospital Heliópolis; 3. Chefe do Serviço de Coloproctologia do Hospital Heliópolis.
\end{abstract}

\begin{abstract}
BATISTA RR; RAMACCIOTTI FILHO PR; CASTRO CAT; ALBUQUERQUE IC; FORMIGA GJS. Síndrome de Fournier Secundária a Adenocarcinoma de Próstata Avançado: Relato de Caso. Rev bras Coloproct, 2010;30(2): 228-231.

RESUMO: A Síndrome de Fournier é uma fasciite necrotizante rapidamente progressiva que acomete a genitália e região perineal. Mesmo com os avanços na terapêutica, a morbidade e a mortalidade desta afecção permanecem elevadas. É relatado caso de paciente masculino, 70 anos, com diagnóstico de adenocarcinoma de próstata avançado. Há um dia com dor e aumento de volume escrotal associado a febre. Ao exame físico, paciente séptico com gangrena gasosa do pênis e escroto; ao toque retal, lesão ulcero-vegetante em parede anterior do reto estendendo-se de 3 a $7 \mathrm{~cm}$ da borda anal. Realizado desbridamento cirúrgico, com identificação de fístula reto-escrotal transtumoral. Estudo histo-patológico da lesão retal confirmou infiltração por adenocarcinoma de próstata. Recebeu alta hospitalar no vigésimo dia de internação, atualmente em acompanhamento oncológico ambulatorial.
\end{abstract}

Descritores: Desbridamento, gangrena de Fournier, neoplasia da próstata, períneo, sepse.

\section{INTRODUÇÃO}

A Síndrome de Fournier é uma fasciite necrotizante rapidamente progressiva, que acomete a genitália e a região perineal. Mesmo com os avanços na terapêutica, a morbidade e a mortalidade desta afecção permanecem elevadas. ${ }^{(1)} \mathrm{O}$ objetivo deste trabalho foi relatar um caso de síndrome de Fournier como complicação do adenocarcinoma de próstata avançado.

\section{RELATO DO CASO}

H.S., masculino, 70 anos, com diagnóstico de adenocarcinoma de próstata avançado, em tratamento com terapia hormonal. Procurou o serviço de emergência com queixa de dor e aumento do volume da bolsa escrotal associado a febre há um dia. Ao exame apresentava sinais de desidratação e sepse. No exame proctológico, à inspeção, períneo de coloração violácea e crepitação a palpação local; o toque retal mostrava lesão úlcero-vegetante em parede anterior do reto, fixa, de consistência pétrea, estendendo-se de 3 a $7 \mathrm{~cm}$ da borda anal. Realizada biópsia da lesão.

Com suspeita diagnóstica de síndrome de Fournier foi realizado correção das alterações de água e eletrólitos, antibioticoterapia com ciprofloxacino $1 \mathrm{~g} /$ dia associado ao metronidazol $1,5 \mathrm{~g} /$ dia e desbridamento cirúrgico. No transoperatório foi diagnosticada fístula complicada do tipo reto-escrotal com extensa área de necrose do escroto, dos testículos e do pênis (figura 1). Realizada orquiectomia bilateral, penectomia total, transversostomia em alça e cistostomia (figura 2).

Trabalho realizado no Serviço de Coloproctologia do Hospital Heliópolis - SP - Brasil. 
O estudo histopatológico e imunohistoquímico da lesão retal confirmaram infiltração por adenocarcinoma de próstata, sendo positiva para PSA, positiva focal para CK20 e negativa para CK7 (figura 3). O paciente apresentou boa evolução pós-operatória e recebeu alta hospitalar no vigésimo dia de internação. Após três meses de seguimento ambulatorial a área operada encontrava-se cicatrizada (figura 4).

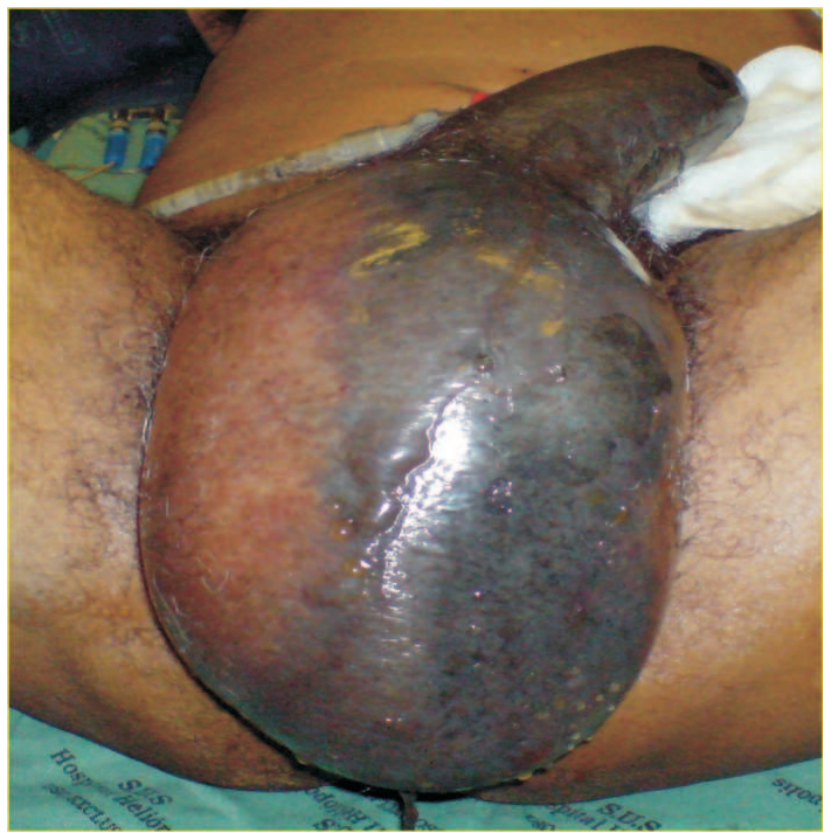

Figura 1 - Necrose do pênis e escroto.

\section{DISCUSSÃO}

Em 1884, Jean Alfred Fournier descreveu cinco casos de fasciite necrotizante de natureza idiopática na região genitoperineal. ${ }^{(2)}$ Atualmente são descritos como fatores de risco para a síndrome de Fournier o abscesso anorretal, diabetes mellitus, alcoolismo, cirrose, desnutrição, obesidade, doença vascular periférica, cirurgias orificiais, vasectomia, trauma perineal, estenose uretral, neoplasia colorretal avançada, neoplasias hematológicas, radioterapia, HIV, apendicite, diverticulite aguda, úlcera duodenal perfurada e doença inflamatória intestinal. ${ }^{(1,3-6)}$

Esta infecção é causada por flora bacteriana de aeróbios e anaeróbios, sendo os principais patógenos Escherichia coli, Bacteroides fragilis, Enterococcus sp. e Clostridium perfringens, ${ }^{(1,3,4)}$ levando a trombose de vasos sanguíneos subcutâneos e consequente necrose da camadas abaixo da pele. ${ }^{(5)}$

O tratamento consiste em suporte hemodinâmico, antibioticoterapia de amplo espectro, desbridamento dos tecidos desvitalizados, oxigenioterapia hiperbárica, investigação do foco primário e, quando necessária, derivação urinária ou fecal. São indicações de cistostomia a estenose uretral ou fonte genitourinária da infecção, enquanto que a colostomia derivativa está indicada na infecção do esfíncter anal ou na presença de grande
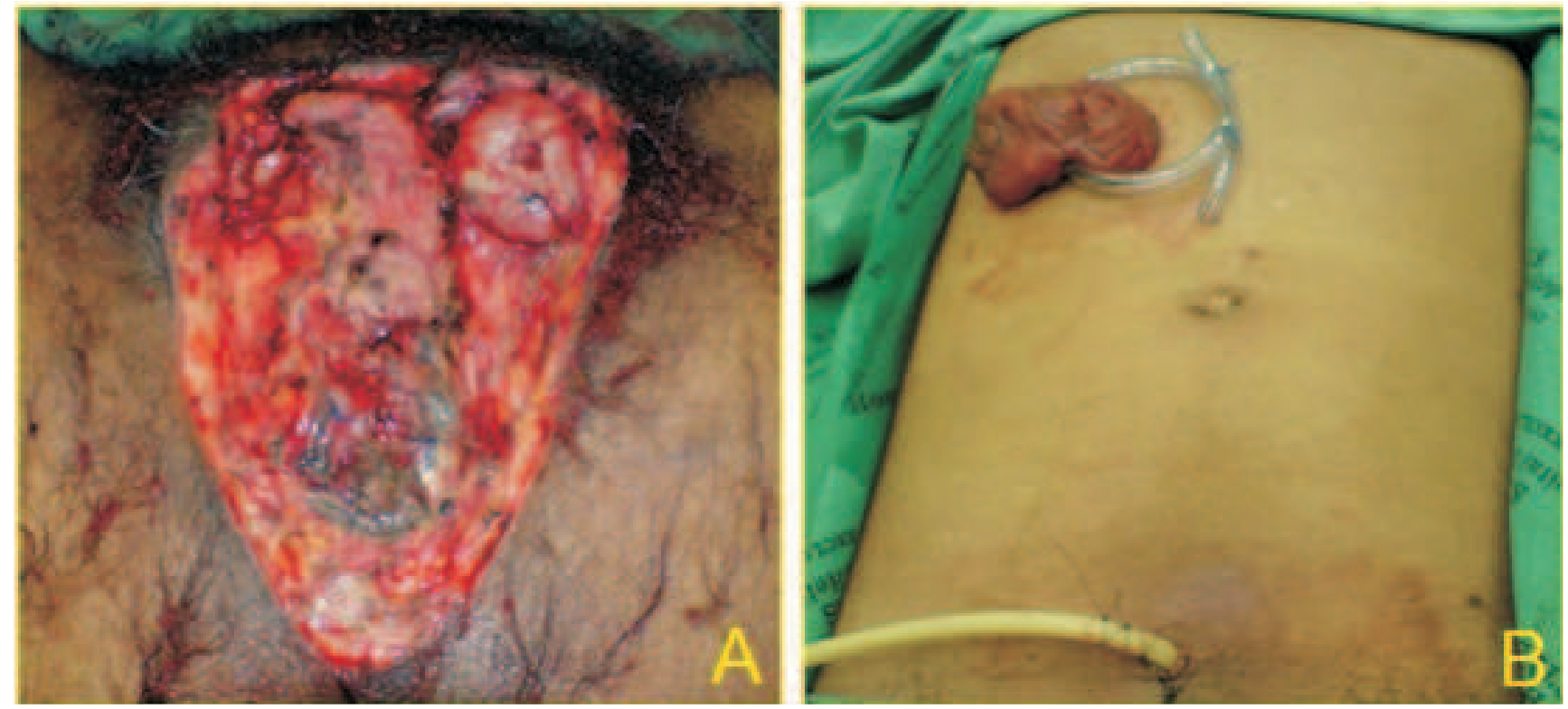

Figura 2 - A: Aspecto final da orquiectomia bilateral com penectomia total; B: Transversostomia e cistostomia. 

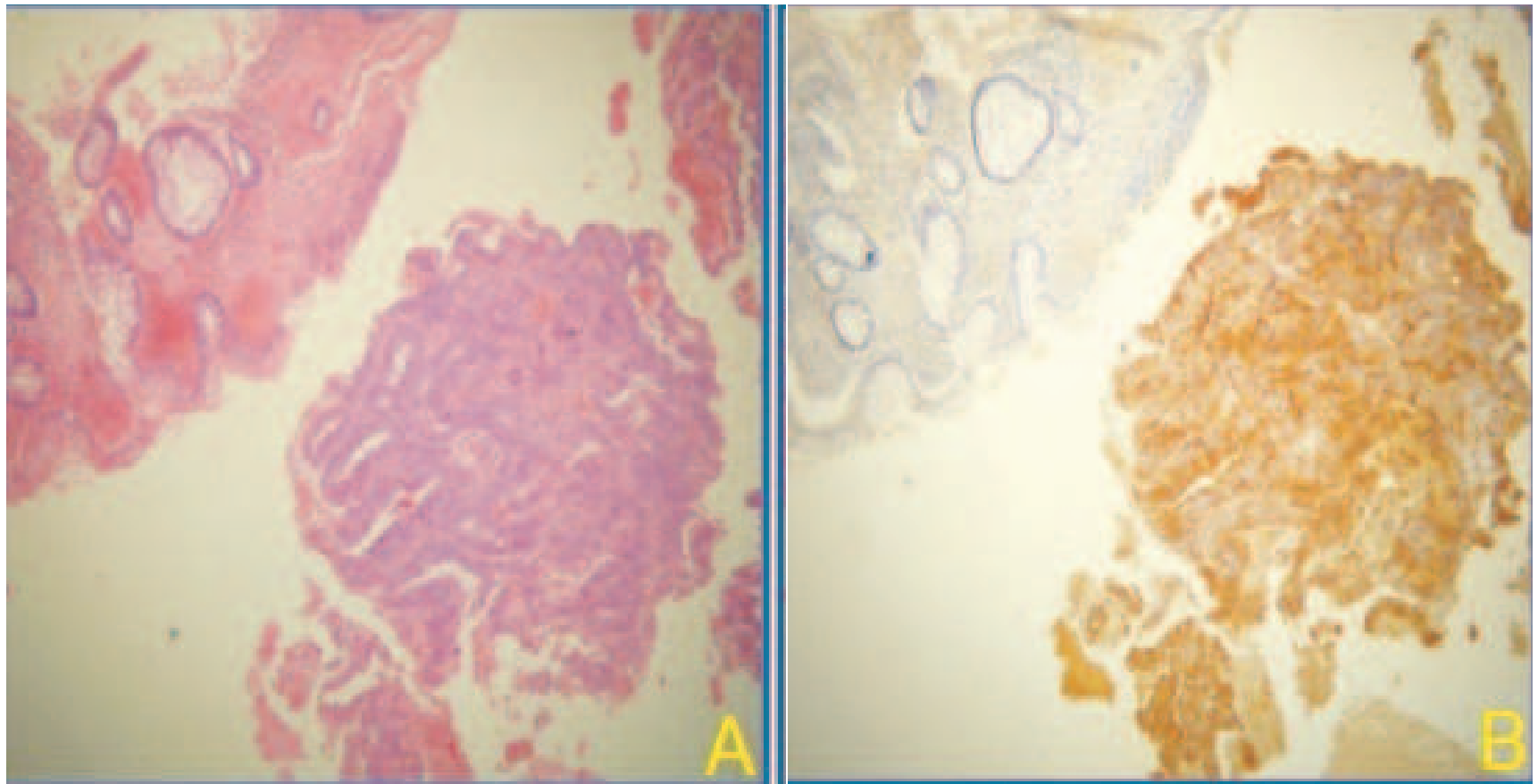

Figura 3 - A: Histologia do tumor retal em $H \&$ E; B: Imunohistoquímica positiva para PSA.

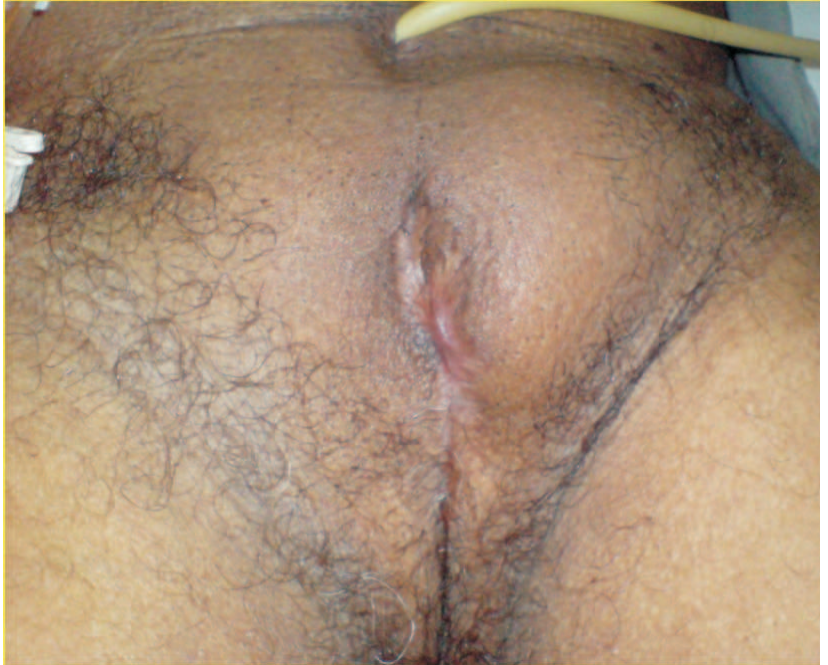

Figura 4-Pós-operatório tardio.

ferida perineal..$^{(1,4,8)}$ A laparoscopia poderá ser usada para diagnosticar causas intra-abdominais de gangrena de Fournier e para realização de colostomia quando necessário. ${ }^{(1)}$

O uso de terapia com oxigênio hiperbárico melhora a sobrevida somente em pacientes com infecção por Clostridium perfringens. No entanto, esta terapia adjuvante pode facilitar a função fagocítica dos macrófagos, promover angiogênese e por isso ajudar na cicatrização. ${ }^{(1,8)}$

Nosso paciente foi submetido a extenso desbridamento com derivação urinária e fecal, devido ao grave quadro clínico de admissão. Apresentou cicatrização satisfatória da ferida operatória apenas com cuidados locais e antibioticoterapia de amplo espectro e, desse modo, não foi necessário o uso de oxigenioterapia hiperbárica.

A síndrome de Fournier é uma afecção agressiva, de rápida evolução, com mortalidade estimada em $20 \%$ a despeito do tratamento adequado. ${ }^{(7)} \mathrm{O}$ diagnóstico e tratamento precoce são a melhor estratégia para otimizar o prognóstico dos pacientes acometidos por esta afecção..$^{(1,6,7)}$

$\mathrm{Na}$ literatura pesquisada não existe descrição da gangrena de Fournier secundária a neoplasia maligna da próstata. $\mathrm{Na}$ ausência de outros fatores de risco, o achado operatório de fístula transtumoral comunicando o reto com base do escroto justifica o quadro descrito. Assim, o adenocarcinoma de próstata avançado deve ser lembrado como um dos diagnósticos etiológicos da síndrome de Fournier. 
Rodrigo Rocha Batista e Cols.

ABSTRACT: Fournier's syndrome is a rapidly progressive necrotizing fasciitis affecting the genitalia and perineal region. Even with the advances in therapy, morbidity and mortality of this disease remain high. We report the case of male patient, 70 years old, diagnosed with advanced adenocarcinoma of the prostate. A day with pain and swelling near scrotum associated with fever. The physical examination revealed septic patient with gas gangrene of the penis and scrotum; on digital rectal examination, a vegetative and ulcerated lesion on the anterior wall of the rectum extending from 3 to $7 \mathrm{~cm}$ from the anal edge. Performed surgical debridement, with identification of scrotal-rectal fistula transtumoral. Histo-pathological study of the lesion confirmed rectal infiltration by adenocarcinoma of prostate. Was discharged from the hospital on the twentieth day of hospitalization, outpatient cancer currently monitoring.

Key words: Debridement, Fournier's gangrene, prostate cancer, perineum, sepsis.

\section{REFERÊNCIAS}

1. Gamagami RA, Mostafavi M, Gamagami A, Lazorthes F. Fournier's gangrene: an unusual presentation for rectal carcinoma. Am J Gastroenterol 1998; 93(4): p 657-658.

2. Fournier AJ. Etude clinique de le gangrene foudroyante de la verge. Semaine Med 1884; 4: p 69.

3. Dewire DM, Bergstein JM. Carcinoma of the sigmoid colon: na unusual cause of Fournier's gangrene. J Urol 1992; 147(3): p 711-712.

4. Gould SWT, Banwell P, Glazer G. Perforated colonic carcinoma presenting as epididimo-orchitis and Fournier's gangrene. Eur J Surg Oncol 1997; 23(4): p 367-368.

5. Bakshi C, Banavali S, Lokeshwar N, Prasad R, Advani S. Clustering of Fournier (male genital) gangrene cases in a pediatric cancer ward. Med Pediatr Oncol 2003; 41(5): p 472-474.

6. Veljkovic R, Milosevic P, Stojanovic S. Fournier's gangrene associated with carcinoma of the colon. Med Pregl 1998; 51(78): p 351-354.
7. Wronski M, Malyszka K, Pawlowski W. Fournier's gangrene in non-resectable colorectal carcinoma. Pol Merkur Lekarski 2004; 17(97): p 62-63.

8. Elgin Y, Demirkasimoglu T, Kucukplakci B, Altundag MB, Altundag K, Misirlioglu $\mathrm{C}$ et al. Anal tumor diagnosed after the recovery of Fournier gangrene. Dig Dis Sci 2006; 51(5): p 889-890.

Endereço para Correspondência:

GALDINO JOSÉ SITONIO FORMIGA

Rua Cônego Xavier, 276

Vila Heliópolis, São Paulo/SP

CEP: 04231-030

Telefone: (11) 2274-7600 (ramal 244)

E-mail: gformiga@ig.com.br 